\title{
High WSS or Low WSS? Complex Interactions of Hemodynamics with Intracranial Aneurysm Initiation, Growth, and Rupture: Toward a Unifying Hypothesis
}

H. Meng, V.M. Tutino, J. Xiang, and A. Siddiqui

\begin{abstract}
SUMMARY: Increasing detection of unruptured intracranial aneurysms, catastrophic outcomes from subarachnoid hemorrhage, and risks and cost of treatment necessitate defining objective predictive parameters of aneurysm rupture risk. Image-based computational fluid dynamics models have suggested associations between hemodynamics and intracranial aneurysm rupture, albeit with conflicting findings regarding wall shear stress. We propose that the "high-versus-low wall shear stress" controversy is a manifestation of the complexity of aneurysm pathophysiology, and both high and low wall shear stress can drive intracranial aneurysm growth and rupture. Low wall shear stress and high oscillatory shear index trigger an inflammatory-cell-mediated pathway, which could be associated with the growth and rupture of large, atherosclerotic aneurysm phenotypes, while high wall shear stress combined with a positive wall shear stress gradient trigger a mural-cell-mediated pathway, which could be associated with the growth and rupture of small or secondary bleb aneurysm phenotypes. This hypothesis correlates disparate intracranial aneurysm pathophysiology with the results of computational fluid dynamics in search of more reliable risk predictors.
\end{abstract}

ABBREVIATIONS: CFD = computational fluid dynamics; $\mathrm{ECM}=$ extracellular matrix; WSS $=$ wall shear stress

l: tracranial aneurysms are pathologic outpouchings of the arterial walls. An estimated $5 \%-8 \%$ of the general population harbors intracranial aneurysms, ${ }^{1}$ though the exact prevalence is unknown because most are asymptomatic. Aneurysm rupture is the most common cause of nontraumatic subarachnoid hemorrhage, a devastating event that carries high rates of mortality, morbidity, and disability, as well as high health care costs. Despite significant improvement in the clinical care of patients with subarachnoid hemorrhage, one-quarter still die, while roughly half of the survivors live with persistent neurologic deficits. ${ }^{2}$ The estimated annual cost for hospitalized patients with unruptured intracranial aneurysms in the United States is $\$ 522,500,000$, and $\$ 1,755,600,000$ for patients with subarachnoid hemorrhage. ${ }^{3}$ Recent advancements and increased use of neurovascular imaging have augmented detection of asymptomatic unruptured intracranial aneurysms, am-

From the Toshiba Stroke and Vascular Research Center (H.M., V.M.T., J.X., A.S.) and Departments of Mechanical and Aerospace Engineering (H.M.), Neurosurgery (H.M., J.X., A.S.), and Biomedical Engineering (H.M., V.M.T.), University at Buffalo, State University of New York, Buffalo, New York.

This work was supported by grants to H.M. from the National Institutes of Health (R01NS064592) and Toshiba Medical Systems.

Please address correspondence to Hui Meng, PhD, Toshiba Stroke and Vascular Research Center, University at Buffalo, Clinical and Translational Research Center, 875 Ellicott St, Buffalo, NY 14203; e-mail: huimeng@buffalo.edu

- Indicates open access to non-subscribers at www.ajnr.org

http://dx.doi.org/10.3174/ajnr.A3558 plifying pressure on clinicians to decide which unruptured aneurysms to treat. This decision is not taken lightly because an overwhelming majority of intracranial aneurysms will not rupture, ${ }^{1}$ while both endovascular and microsurgical treatments carry the risk of associated morbidity and mortality. Consequently, there is a real need for objective aneurysm rupture risk assessments that could reliably predict those at highest risk and subsequently select only them for intervention.

To this end, investigators have tried to identify aneurysmal characteristics that are associated with intracranial aneurysm growth and rupture. Hemodynamics is one of most widely accepted factors contributing to aneurysm pathophysiology, playing a fundamental role in the mechanisms of initiation, growth, and rupture. ${ }^{4-9}$ Recent studies using image-based computational fluid dynamics (CFD) modeling and statistical analyses have identified connections between the hemodynamic properties of intracranial aneurysms and the likelihood of their growth and rupture. ${ }^{5,9}$ Such findings have highlighted the exciting possibility that aneurysmal hemodynamics may provide objective metrics to improve rupture risk stratification.

\section{The "High-versus-Low WSS" Controversy}

A number of engineering and computational researchers have published CFD studies associating specific hemodynamic parameters with intracranial aneurysm growth and rupture. ${ }^{5,7-14}$ While 


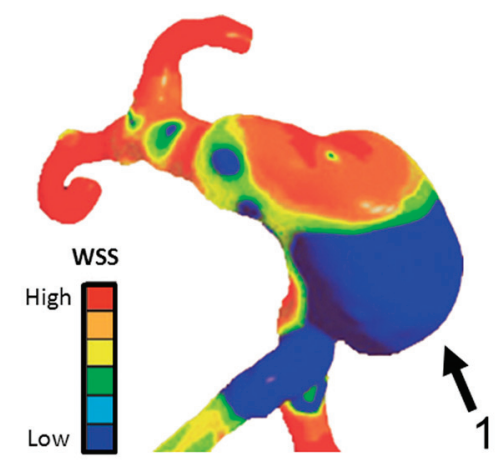

A

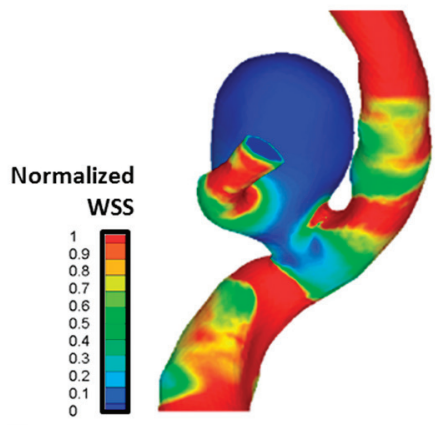

C

FIG 1. Examples of aneurysm growth $(A$ and $B)$ and rupture $(C$ and $D)$ correlated with high wall shear stress and low WSS. $A$, In a serial study consisting of a baseline and 4 follow-ups, AcevedoBolton et $\mathrm{al}^{10}$ found that the maximal growth region (1) of a giant basilar fusiform intracranial aneurysm consistently had the lowest WSS. B, Sugiyama et $\mathrm{al}^{13}$ reported 2 adjacent growing aneurysms, with different hemodynamic characteristics and growing patterns in 1 patient. The proximal growing aneurysm (2) was subjected to local high flow in the growing lobe, while the distal aneurysm (3) was associated with low and oscillatory WSS in the entire aneurysm sac. C, From multivariate statistical analysis of 119 aneurysms, Xiang et a $\left.\right|^{5}$ found that intracranial aneurysm rupture could be predicted by low WSS and high oscillatory shear index. D, Castro et a ${ }^{11}$ found, from analysis of 26 aneurysms, that intracranial aneurysm rupture was correlated with high maximum WSS. Images were adapted with permission from the cited references.

clinicians welcome such effort, the growing number of proposed parameters remains inconsistent and confusing. ${ }^{15-17}$ The most highlighted and controversial parameter has been wall shear stress (WSS), the frictional force exerted by the flowing blood tangentially on the vessel lumen. Both high and low aneurysmal WSS have been separately correlated with intracranial aneurysm growth and rupture. ${ }^{16}$ This controversy is highlighted by findings presented in Fig 1.

Presently, it is unclear whether the "high-versus-low WSS" controversy stems purely from study limitations, such as skewness due to small sample sizes, inconsistent parameter definitions, flawed experimental design, variability in assumptions and compromises in CFD, or from the inherent complexity and heterogeneity of intracranial aneurysm growth and rupture mechanisms. ${ }^{16,17}$ How high WSS and low WSS might be involved in aneurysm development remains unclear because the biologic mechanisms underlying growth and rupture and their interaction with hemodynamics have not been clearly elucidated. In this review, we provide a novel view of aneurysm development and a unified hypothesis regarding the mechanistic role of both high and low WSS in intracranial aneurysm growth and rupture.

\section{Relationship between Hemodynamics and Aneurysm Development}

Aneurysm Development Is a 3-Way Interactive Process Driven by Hemodynamics. In clinical practice, aneurysmal geometry (especially size and aspect ratio) has been the principal parameter used to gauge the rupture likelihood of intracranial aneurysms. ${ }^{18}$ However, hemodynamics provides mechanical triggers that are transduced into biologic signals leading to this geometric evolution. Aneurysmal geometry and hemodynamics are mutually causal: Geometry instantaneously determines flow conditions, while flow drives aneurysm remodeling/growth through pathobiology, thereby determining future geometry (ie, enlargement and shape change). As this process continues, an intracranial aneurysm will either grow until homeostasis (stability) is reached or until its wall strength can no longer withstand the hemodynamic stress, in which case rupture occurs. This is best illuminated by a triangular relationship among geometry, flow, and pathobiology (Fig 2A).

As illustrated in Fig $2 A$, hemodynamics interacts with the aneurysm wall through blood flow (WSS and blood pressure). Pressure elicits tensile stresses in the wall, which are felt by vascular mural cells, namely smooth muscle cells and fibroblasts. Under unbalanced stresses, these mural cells can regulate collagen $\mathrm{dy}$ namics by cross-linking and synthesizing new collagen and degrading old collagen. ${ }^{19}$ Meanwhile, endothelial cells lining the vessel lumen sense changes in WSS from blood flow and transduce these mechanical signals into biologic signals, activating pathways to maintain vascular homeostasis. ${ }^{20}$ Through endothelial cell-mediated biology, WSS not only regulates vascular tone but also drives vascular remodeling under sustained deviations from physiologic baselines. ${ }^{19}$ Pathologically high or low WSS and certain spatiotemporal patterns of its variation can potentiate endothelial cells to pathologic responses and aberrant functions. Presently, it is known that abnormal WSS drives endotheliummediated proinflammatory responses, ${ }^{21}$ matrix metalloproteinase activation, ${ }^{22}$ cell death, ${ }^{23}$ extracellular matrix (ECM) degradation, and vascular remodeling. ${ }^{4,24,25}$

Hemodynamics Plays a Critical Role in Intracranial Aneurysm Pathogenesis. A cerebral aneurysm is defined as a local outpouching of an intracranial artery exhibiting internal elastic lamina loss, tunica media thinning, and ECM degradation ${ }^{23}$ and can either be saccular or fusiform. The pathogenesis of fusiform aneurysms, with some exceptions, ${ }^{26}$ is closely related to atherosclerosis. ${ }^{27}$ Because a 

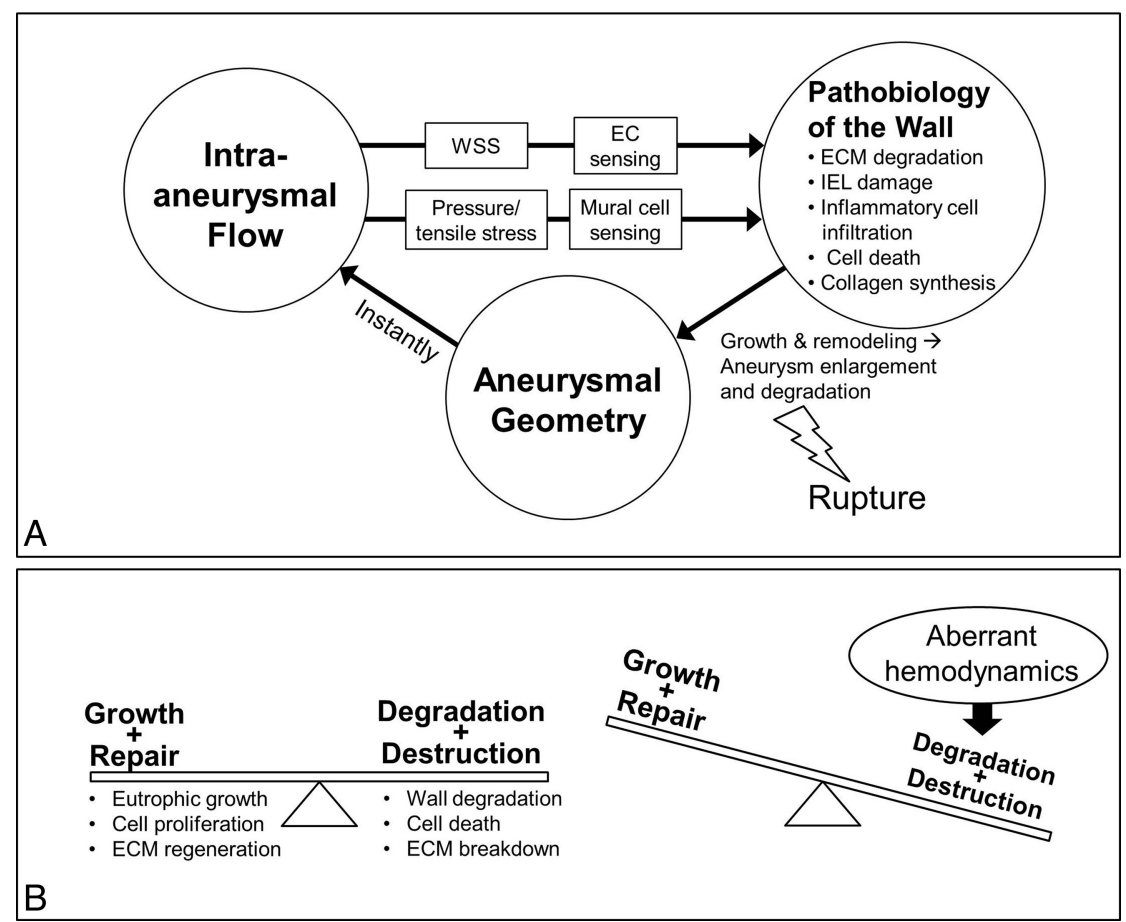

FIG 2. How hemodynamics plays into intracranial aneurysm dynamics. A, Three-way relationship of aneurysmal geometry, flow, and pathobiology. Flow exerts mechanical forces on the vessel wall, eliciting cell-mediated biologic pathways. Sustained changes in flow (thus WSS and pressure) lead to remodeling of the wall, resulting in aneurysm geometry change. In turn, geometry determines the flow, which could drive further biologic processes. $B$, The balance between growth/repair and degradation/destruction results in aneurysm stability. Aberrant hemodynamics disrupts the balance and amplifies degradation and destruction mechanisms, leading to enlargement and rupture of aneurysm.

vast majority $(>80 \%)$ of intracranial aneurysms are saccular, ${ }^{28}$ we will focus our discussion on this type of aneurysm.

The natural history of saccular intracranial aneurysms consists of 3 phases: initiation, growth, and either stabilization or rupture, with only a small minority of aneurysms ever progressing to rupture. ${ }^{1}$ Intracranial aneurysm formation is the result of the interaction between the arterial wall and hemodynamic forces. ${ }^{29}$ The cerebral vasculature is intrinsically prone to the effects of hemodynamic forces due to the lack of external elastic lamina, medial elastin, and supporting adventitial and perivascular tissues. ${ }^{30,31}$ Moreover, cerebral arteries display structural irregularities at bifurcation apices, typical sites for saccular intracranial aneurysms. ${ }^{32}$ These factors make such locations prone to insults by hemodynamic stresses and subsequent internal elastic lamina damage and aneurysm formation. ${ }^{33}$

Recent studies in animal models have elucidated the pivotal role of hemodynamics in intracranial aneurysm initiation. ${ }^{25,34-38}$ Flow acceleration adjacent to impingement points at bifurcations produces a complex hemodynamic environment of high WSS and a positive WSS gradient along the flow, ${ }^{4,37}$ which, through endothelium-mediated mechanotransduction, can initiate cascades of biochemical signals within the vessel wall and trigger aneurysm initiation. ${ }^{4,25,39}$ In a hemodynamics-only model of intracranial aneurysm initiation, Metaxa et $\mathrm{al}^{4}$ demonstrated that aneurysm initiation occurs when high WSS and positive WSS gradient exceed a certain threshold. This insult leads to local internal elastic lamina loss, media thinning, and bulge formation, ${ }^{4,25,36}$ the earliest signs of intracranial aneurysm formation.
Prior to the initial aneurysmal damage triggered by hemodynamics, the cerebral vasculature may have already been weakened by various acquired (eg, cigarette smoking and hypertension $)^{40,41}$ or inherited risk factors (eg, polycystic kidney disease). ${ }^{1}$ These factors compromise the ability of the cerebral vasculature to tolerate and properly adapt to hemodynamic insult, ${ }^{39}$ most likely by lowering the threshold for the onset of pathologic responses. ${ }^{42,43}$ Certainly, variations in hemodynamics and different risk factors among different individuals contribute to the heterogeneity of the disease.

Many studies have tried to incorporate various aneurysm risk factors such as hypertension, decreased collagen crosslinking, and estrogen deficiency into animal models. ${ }^{34,44,45}$ In general, these studies have found that aneurysm progression starts with initial endothelial cell responses ${ }^{45}$ and smooth muscle cell phenotypic modulation, after which an escalating inflammatory response may be provoked, accompanied by ECM remodeling and degradation and cell death. ${ }^{46}$ These studies demonstrated that after intracranial aneurysm initiation, inflammation may play an important role in aneurysm progression (eg, inflammatory infiltrates produce matrix metalloproteinases, leading to wall degradation). ${ }^{34}$ These animal models demonstrated aneurysm development only when a hemodynamic insult was also applied. This reaffirms the conclusion that hemodynamic insult is necessary for intracranial aneurysm genesis.

Aberrant Hemodynamics Can Disrupt Balance and Drive Intracranial Aneurysm Growth and Rupture. Aneurysm growth is dictated by the interplay between the local hemodynamic-biomechanical environment and aneurysm pathobiology. In the aneurysm wall, there are coincident and concurrent eutrophic changes (cell proliferation and ECM production) and destructive changes (cell death and ECM degradation) ongoing throughout the natural history of the intracranial aneurysm (Fig $2 B) .{ }^{23}$ When these 2 processes are balanced, the intracranial aneurysm remains stable; when the balance is disrupted, it may rupture. Clearly, aneurysm growth and rupture requires a disruptive agent. We believe that aberrant hemodynamics, chiefly through abnormal WSS, is a major disruptive agent. As illustrated in Fig $2 B$, intracranial aneurysm growth and rupture occur when aberrant hemodynamics cause destructive changes to outweigh eutrophic changes, making the aneurysm wall increasingly weaker and prone to rupture. ${ }^{23}$

Different Manifestations of Intracranial Aneurysms. Intracranial aneurysm lesion presentation is highly heterogeneous in almost every observable metric (Fig 3). Three principal aneurysm pheno- 

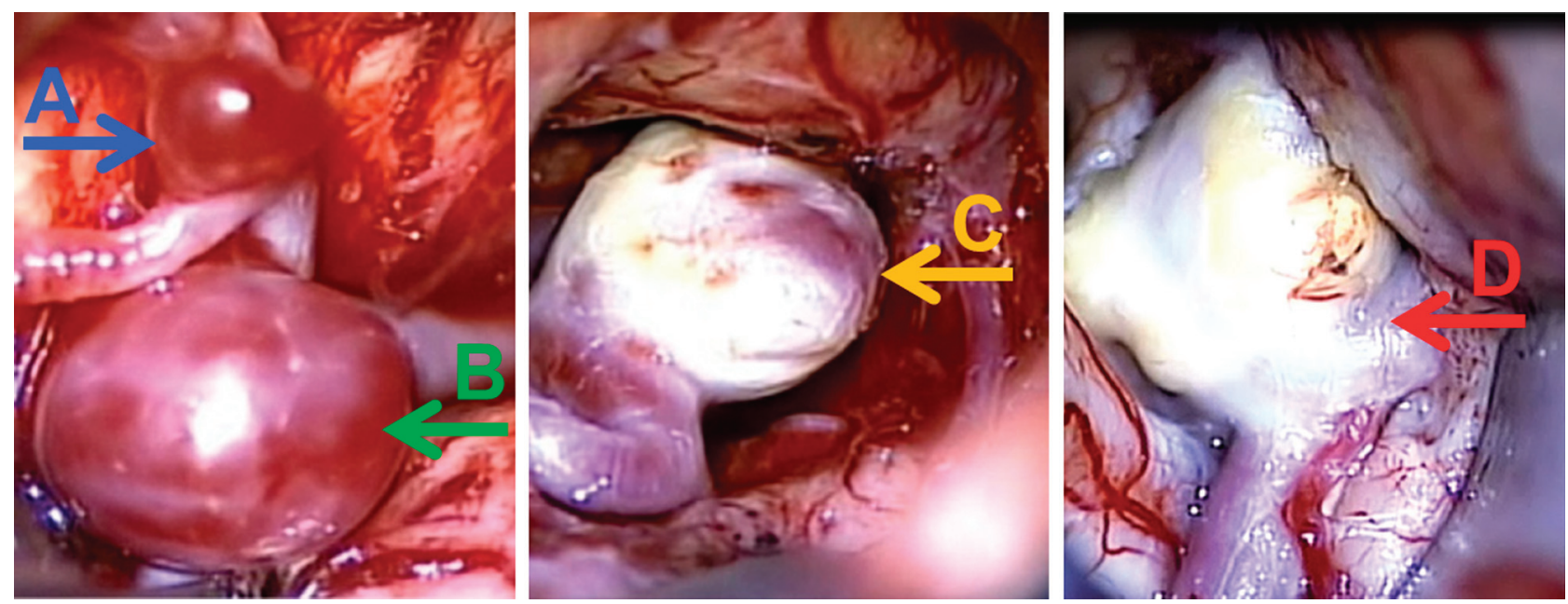

FIG 3. Heterogeneity of saccular aneurysms as a spectrum from type I $(A)$ to type II $(D)$ with various intermediate or combination types in between ( $B$ and $C$ ). A, A small thin-walled, entirely translucent aneurysm (type I). $B, A$ mostly thin-walled aneurysm with some thicker-walled patches. C, A mostly thick-walled aneurysm with a few thin-walled patches. D, An aneurysm with an entirely thick, atherosclerotic wall (Type II). Images adapted with permission from Kadasi et al $2012^{50}$

types have been reported from intraoperative observation of unruptured intracranial aneurysms. ${ }^{47-49}$

The first types are small aneurysms $(<4 \mathrm{~mm})$ with uniformly thin, smooth, hypocellular, translucent walls, through which reddish blood flow can be visualized at the time of surgical clipping. We refer to these as type I aneurysms (an example is seen in Fig $3 A$ ). The second types are entirely thickwalled large aneurysms $(>10 \mathrm{~mm})$, with an irregular surface on which whitish/yellowish atherosclerotic plaques obstruct the visualization of blood. We refer to these as type II aneurysms (an example is seen in Fig 3D). The third types are medium-sized aneurysms with a combination of thin- and thickwalled characteristics in different regions ${ }^{47,49}$ or with intermediate wall thickness. ${ }^{50}$ We refer to these as a combination type (examples are seen in Fig $3 B,-C$ ). According to Kadasi et $a l,{ }^{50}$ the distribution of these 3 phenotypes in unruptured aneurysms is $27 \%, 8 \%$, and $65 \%$ for types I and II and the combination type, respectively.

Histologic analyses of both unruptured and ruptured aneurysm specimens from autopsy studies have mirrored such phenotypical classification: ${ }^{50-54}$ Small intracranial aneurysms $(<10$ $\mathrm{mm}$ ) have a higher rate (48\%) of having thin, transparent, and hypocellular walls; absent smooth muscle cells, and inflammatory cells. ${ }^{53,54}$ Large intracranial aneurysms $(>10 \mathrm{~mm})$ have a low rate $(6 \%)$ of thin-walled regions but a high rate of thick walls with atherosclerotic changes, proliferation of smooth muscle cells, and inflammatory cells. ${ }^{51-54}$

These data suggest that intracranial aneurysm phenotypes exist on a spectrum: At one extreme is the small thin-walled phenotype (type I); at the other extreme is the large thick-walled phenotype (type II); and in between is a continuum representing an amalgamation of these 2 basic types. The recognition of different phenotypes in both incidentally discovered and ruptured aneurysms suggests that there may be a variety of nonconvergent hemodynamic-biologic pathways involved in the natural history of intracranial aneurysms.
Rupture Potential versus Rupture Event. Certainly, intracranial aneurysm rupture has been associated with intense physical activities and accompanying high blood pressure. ${ }^{55}$ Large elevations in mean arterial blood pressure during such activities can increase aneurysm wall tension to a level that leads to rupture. ${ }^{55}$ An increased heart rate during physical exertion and emotional excitement have also been suspected of contributing to rupture, ${ }^{56}$ possibly through the increased frequency of cyclic stretching, which leads to hastened fatiguing of the wall. ${ }^{57}$

Why only some aneurysms are subjected to such catastrophe through routine activities is related to the vulnerable condition of their wall. Before rupture, the wall may have biologically remodeled and deteriorated with time, ${ }^{33}$ decreasing the wall strength to such a level that normal physical exertion could generate enough pressure to push the wall tensile stress over the limit (ie, exceeding the wall strength), ${ }^{33}$ rupturing the wall. Therefore, while the rupture episode itself is triggered by temporary pressure and/or frequency surge and wall failure, the predisposition of an intracranial aneurysm wall to rupture is due to biologic degradation, mediated by the interaction between hemodynamics and pathobiology with time.

\section{A Unifying Hypothesis}

Understanding the nature and origin of the high-versus-low WSS controversy is important in developing quantitative guidance for physicians to make better intracranial aneurysm treatment decisions. ${ }^{15,16}$ Currently, the source of such heterogeneous findings remains elusive. ${ }^{16,17}$ Here, we speculate that this heterogeneity may reflect the inherent complexity of natural history of intracranial aneurysms and the diversity of growth and rupture mechanisms. Understanding the biologic processes activated by different hemodynamic conditions, such as high and low WSS, may shed light on possible mechanisms that lead to intracranial aneurysm growth and rupture.

To address the conflicting CFD findings, we draw on the current understanding of hemodynamically induced pathobiologic 
Vascular responses to aberrant WSS conditions reported in literature

\begin{tabular}{|c|c|}
\hline $\begin{array}{c}\text { Pathobiologic Responses } \\
\text { to High WSS and Positive } \\
\text { WSS Gradient }^{\mathrm{a}}\end{array}$ & $\begin{array}{c}\text { Pathobiologic Responses to Low } \\
\text { WSS and High OSI }\end{array}$ \\
\hline EC damage $\mathrm{e}^{24,45}$ & $\begin{array}{l}\text { Proinflammatory ECs that are } \\
\text { "leaky" and "sticky"21,62 }\end{array}$ \\
\hline EC turnover ${ }^{25,72}$ & Increased $\operatorname{ROS}^{62}$ \\
\hline $\begin{array}{l}\text { MMP production by mural } \\
\text { cells }^{25}\end{array}$ & $\begin{array}{l}\text { Increased inflammatory cell } \\
\text { infiltration }\end{array}$ \\
\hline ECM degradation $^{36}$ & MMP production by macrophages ${ }^{61}$ \\
\hline Medial thinning ${ }^{36}$ & SMC proliferation and migration ${ }^{63}$ \\
\hline Mural cell apoptosis ${ }^{25}$ & Thrombus formation ${ }^{23,63}$ \\
\hline
\end{tabular}

Note:-EC indicates endothelial cell; ROS, reactive oxygen species; MMP, matrix metalloproteinase; OSI, oscillatory shear index; SMC, smooth muscle cell.

${ }^{a}$ From the literature on intracranial aneurysm genesis.

${ }^{\mathrm{b}}$ From the literature on atherogenesis.

responses in better described vascular pathologies in the literature (the pathogenesis of atherosclerosis and intracranial aneurysm). Such knowledge, summarized in the Table, helps us conceptualize possible roles of similar responses in intracranial aneurysm pathophysiology.

High and Low WSS Can Drive Different Mechanistic Pathways of Intracranial Aneurysm Growth and Rupture. We submit that aberrant hemodynamics of both low and high WSS can drive intracranial aneurysm growth and rupture via different biologic mechanisms:

1) Low WSS and a high oscillatory shear index can trigger inflammatory-cell-mediated destructive remodeling.

2) High WSS and a positive WSS gradient can trigger muralcell-mediated destructive remodeling.

By the pathobiologic effects in the Table, abnormal hemodynamic conditions of high and low WSS can disrupt the equilibrium between eutrophic and degradative processes in the intracranial aneurysm wall (Fig 2B). ${ }^{23}$ Specifically, both high and low WSS can incite proteolytic and oxidative damage, which causes ECM degradation and cell death, thereby facilitating aneurysm growth and rupture. This hypothesis lays the foundation for a conceptual framework that helps us begin to dissect the complexity of the hemodynamics-aneurysm pathophysiologic interaction.

Figure 4 illustrates the unified role of hemodynamics throughout aneurysm development. Initiation of intracranial aneurysms is induced by a high WSS and a positive WSS gradient. ${ }^{4,37}$ Through endothelial cell mechanotransduction, these hemodynamic stresses initiate biochemical cascades when they exceed certain thresholds, leading to local production and activation of proteases (most important, matrix metalloproteinase -2 and -9) by mural cells, ${ }^{25}$ massive internal elastic lamina damage, ${ }^{4}$ and apoptosis, ${ }^{25}$ which are responsible for media thinning and bulge formation. ${ }^{37}$ Most interesting, inflammatory cell infiltration was not observed in early-stage intracranial aneurysm initiation, ${ }^{25}$ and macrophage depletion did not attenuate aneurysm formation, indicating that hemodynamic initiation of intracranial aneurysms is not mediated by infiltrating inflammatory cells. (M. Mandelbaum, J. Kolega, J.M. Dolan, A. Siddiqui, H. Meng, December 2012, unpublished data).

After initiation, aneurysmal bulge enlargement typically exposes the sac to increasingly lower WSS, leading to the biologic pathway shown by the right branch in Fig 4. After a recirculation zone forms in the sac, the flow environment is likely to be dominated by low and oscillating WSS. This condition is exacerbated if secondary vortices form and/or flow instability increases. ${ }^{58}$ Low and oscillatory shear stress is known to elicit an inflammatory response in the endothelium. Endothelial cells produce reactive oxygen species, and upregulate surface adhesion molecules and cytokines in the vessel wall and increase luminal permeability. ${ }^{59,60}$ A "sticky" and "leaky" endothelium, in combination with an increased blood residence time, facilitates leukocyte transmigration into the wall during aneurysm development. These inflammatory infiltrates can massively produce matrix metalloproteinases to degrade the $\mathrm{ECM},{ }^{61}$ thus tipping the balance between eutrophic and degradative processes and driving intracranial aneurysm growth and rupture. ${ }^{23}$ Furthermore, such "disturbed flow" environments also promote the formation of atherosclerotic plaques, ${ }^{23,62}$ which exacerbate the effects of inflammatory cells. The inflammatorycell-mediated degradation becomes even more pronounced upon the formation of a luminal thrombus, which can further trap macrophages and neutrophils, and harbor proteases, reactive oxygen species, and oxidized low-density lipoproteins. ${ }^{63}$ Because wall degradation via this pathway relies on leukocyte infiltration, we term it "inflammatory-cell-mediated destructive remodeling."

On the other hand, impinging flow may persist after bulge formation in some aneurysms, so that high WSS and positive WSS gradient could remain prevalent in the aneurysmal sac. For example, in intracranial aneurysms with high-curvature parent vessels, ${ }^{64}$ high aneurysm angle, ${ }^{65}$ or high inflow angle, ${ }^{66}$ inflow from the parent vessel can carry high inertia and impinge on the wall. This hemodynamic condition could lead to the pathway shown by the left branch in Fig 4. Contrary to the right branch, mural cells, instead of inflammatory cells, are most likely responsible for the destructive changes in the intracranial aneurysm wall in this pathway, just as in initiation. ${ }^{25}$ Certainly, the high WSS environment is not conducive to leukocyte infiltration, which requires sufficient blood residence time and the endothelial cell responses commonly elicited under low WSS and oscillatory flow. ${ }^{62,67}$ Systemic depletion of macrophages does not suppress aneurysm development induced by high WSS. Rather, in this pathway, phenotype-modulated smooth muscle cells are the source of proteolytic activities. ${ }^{25}$ Therefore, we term it "mural-cell-mediated destructive remodeling."

Although leukocyte infiltration is not thought to be involved in the high-WSS-driven pathway, proinflammatory behavior could still be playing a critical role. Recently, it was found that in the internal elastic lamina damaged zones during intracranial aneurysm genesis, smooth muscle cells upregulated the proinflammatory proteins monocyte chemoattractant protein-1 and the transcription factor nuclear factor- $\kappa \mathrm{B}$, (M. Mandelbaum, J. Kolega, J.M. Dolan, A. Siddiqui, H. Meng, December 2012, unpublished data) and produced matrix metalloproteinase -2 and -9 , which are required for aneurysmal degradation. ${ }^{25}$ These "inflammatory" smooth muscle cells lose some of their contractile phenotype by decreasing smooth muscle actin expression. In some ways, smooth muscle cells under high WSS conditions could act like inflammatory cells to cause aneurysmal remodeling.

While some intracranial aneurysms may be dominated by one pathway, others could switch from one to another as the geometry 


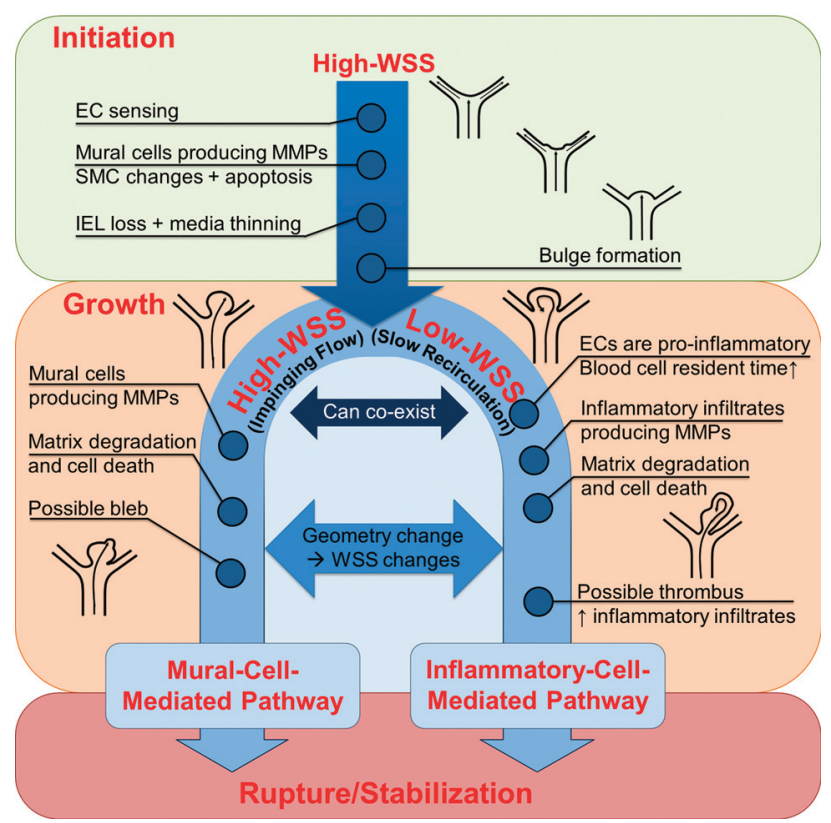

FIG 4. A unified role of high and low WSS in aneurysm initiation, growth, and rupture.

changes (eg, during the initiation of blebs), as illustrated by the double-headed arrows between the branches in Fig 4. In some aneurysms, both biologic mechanisms might dominate different parts of the sac, depending on the local hemodynamic condition. The 3-way relationship among flow, pathobiology, and geometry (Fig 2A) can lead to further complexities in intracranial aneurysm pathophysiology, both longitudinally (at different time points) and cross-sectionally (at different spatial regions of the aneurysm). As these conditions change, so does the balance between eutrophic and destructive processes, resulting in either stabilization or rupture of the intracranial aneurysm (Fig $2 B$ ).

Taken together, high WSS and low WSS are 2 aberrant hemodynamic conditions that could elicit pathologic remodeling pathways to drive intracranial aneurysm growth and rupture. Recognizing the heterogeneity of aneurysm phenotypes (see "Different Manifestations of Intracranial Aneurysms"), one cannot help but ask if these 2 pathways might be responsible for the 2 basic phenotypes (type I and type II) and if their interchange might account for the wide spectrum in between. We suspect that they do.

Emergence of Different Intracranial Aneurysm Phenotypes. We further hypothesize that the high-WSS-driven, mural-cell-mediated pathway is responsible for type I aneurysms, while the lowWSS-driven inflammatory-cell-mediated pathway is responsible for type II aneurysms. The interplay between these 2 pathways, both longitudinally and cross-sectionally, contributes to the wide spectrum of combined intracranial aneurysm phenotypes. This concept is illustrated in Fig 5.

Small and transparent type I aneurysms develop relatively quickly. ${ }^{68}$ Their walls lack inflammatory cells and other cell types, as demonstrated by intraoperative $\mathrm{e}^{4-49}$ and postmortem studies. ${ }^{52-54}$ It is possible that they are formed by impinging flow through the high-WSS-driven degradation mechanisms, as illustrated on the left side of Fig 5. The resident mural cells produce matrix maetalloproteinases under high WSS conditions, leading to significant ECM degradation and cell death by anoikis (apoptosis due to loss of ECM anchorage). ${ }^{69}$ This degradative process may deplete the sac of resident cells and elastin fibers and reduce collagen fibers, forcing the remaining ECM to stretch, creating a stiff thin wall. ${ }^{19,54}$

On the other hand, the large, thick-walled, atherosclerotic type II aneurysms appear to have developed during a longer period of time through various attempts to heal the sac. ${ }^{47-49}$ Indeed, most inflammatory cells found in aneurysm specimens came from this type of aneurysm. ${ }^{52-54}$ We therefore conjecture that the natural history of type II aneurysms (after initiation) is dominated by the inflammatory-cell-mediated pathway, as illustrated on the right side of Fig 5. The destructive remodeling here is accompanied by increased inflammatory cell infiltration and smooth muscle cell proliferation, especially after atherosclerotic plaque and/or thrombus formation. ${ }^{63,70}$ These processes lead to large, atherosclerotic, and thrombotic intracranial aneurysm phenotypes.

We expect that both pathways can dominate different phases of intracranial aneurysm natural history and/or different regions of the growing aneurysm, thereby contributing to the wide spectrum of the combined type aneurysms. As the aneurysm geometry changes, so does the dominant flow condition (jet or recirculation) and the pathway it espouses.

\section{SUMMARY}

Aneurysms occurring in different locations and perienvironments, with varied morphologies and flow dynamics, are associated with complex genetic and environmental contributing factors, which could modify the vascular response to hemodynamics. As such, studies containing a limited cohort of intracranial aneurysms are inevitably based on skewed samples. It is not surprising that their findings sometimes do not converge. Additionally, conflicting CFD results have been rationalized by inconsistent parameter definitions, flawed experimental design, or variability in assumptions and compromises adapted in CFD simulations.

\section{A. Hemodynamic-Biological Mechanistic Pathways}

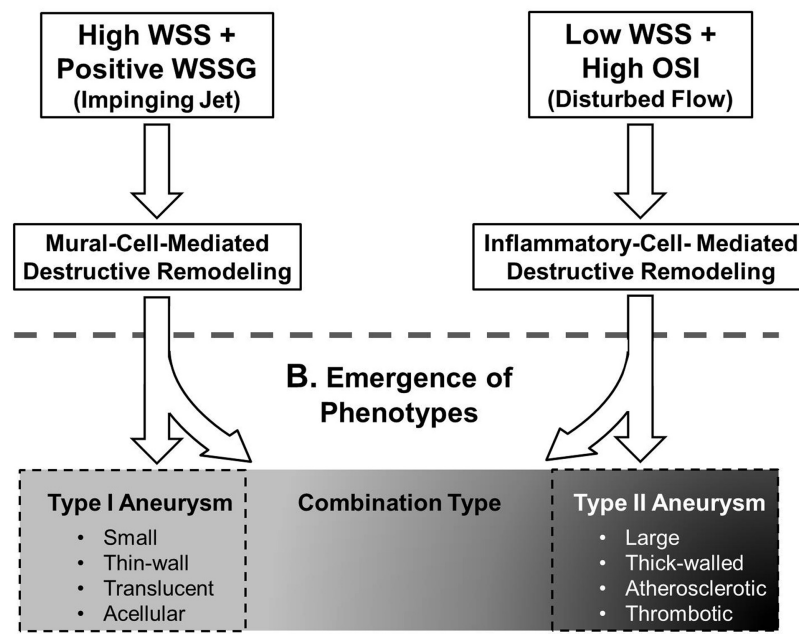

FIG 5. Two hypothesized, independent, hemodynamic-biologic pathways that drive intracranial aneurysm growth and rupture $(A)$ and the proposed relationship between them and the spectrum of intracranial aneurysm phenotypes $(B)$. 
These factors aside, we believe there is intrinsic mechanistic complexity concerning intracranial aneurysms, which underlies the high-versus-low WSS controversy.

We submit that inconsistent findings about the role of WSS are principally a result of the inherent heterogeneity in intracranial aneurysm natural history and the diversity of hemodynamically driven growth and rupture mechanisms. A novel concept can help delineate such complexity: Aberrant hemodynamics including both high WSS and low WSS can tip the balance that maintains vascular homeostasis and can drive destructive remodeling to cause intracranial aneurysm progression and rupture. We propose that 2 independent hemodynamically driven biologic pathways could be associated with intracranial aneurysm growth and rupture: an inflammatory-cell-mediated pathway that is induced by low WSS and a high oscillatory shear index, and a muralcell-mediated pathway that is induced by high WSS and a positive WSS gradient. Furthermore, these 2 hemodynamic-biologic pathways may be responsible for the 2 aneurysm phenotypes: large thick-walled atherosclerotic aneurysms (type II) and smaller thin-walled translucent aneurysms (type I), respectively. These processes reflect the variations in the tripartite interactions of geometry, flow, and pathobiology, which become apparent in the spectrum of intracranial aneurysm phenotypes. Each phenotype attempts to re-establish homeostasis between the eutrophic and destructive forces. If homeostasis is achieved, the aneurysm stabilizes; if not, it ruptures.

We argue that the high-versus-low WSS controversy is a manifestation of the heterogeneity in intracranial aneurysm pathophysiology and its intricate relationship with hemodynamics.

\section{Future Directions}

Going forward, we expect that large, multicenter, global studies will be needed to obtain a more comprehensive picture of intracranial aneurysm hemodynamic pathophysiology and to develop more reliable risk-prediction models. This effort will likely require better classification of aneurysms (eg, based on aneurysm size, location, phenotype, perienvironment, and patient population), rather than treating them as a conglomeration. Different predictive models could be extracted from different classes of datasets and applied to intracranial aneurysms that belong to specific categories. For example, statistical analyses of small aneurysms may produce a different set of predictive parameters (related to high WSS and a positive WSS gradient) from that of large aneurysms (related to low WSS and a high oscillatory shear index). We envision that intracranial aneurysm cases could be carefully classified and then subjected to the appropriate predictive models. The models should reflect the underlying mechanisms driving aneurysm growth and rupture. As such, intracranial aneurysm classification based on size alone may not be highly accurate. An alternative strategy would be to identify type I and II aneurysms from imaging and then to perform subgroup risk analyses and management.

There is a need to study the role of hemodynamic-biologic interactions in the natural history and rupture propensity of intracranial aneurysms in experimental models. Animal models commonly used for clinical studies are good for testing medical devices and investigating hemodynamics ${ }^{71}$ but are generally biologically deficient. On the other hand, current endogenous models $^{34,37}$ capture some hemodynamic-biologic effects in the early stages of aneurysm formation but lack the ability to study growth and rupture. Therefore, improved animal models, aided by advancements in in vivo and molecular imaging, are needed to elucidate the hemodynamic-biologic mechanisms driving aneurysm growth, and models of aneurysm rupture must be developed to study the hemodynamic and biologic mechanisms involved in rupture.

Patient-specific CFD studies must be complemented by hemodynamic-biologic mechanistic studies because these 2 approaches are mutually informative and beneficial. Analysis of patient-specific CFD results may generate new questions concerning the pathophysiology of intracranial aneurysm (ie, which mechanistic studies should be investigated). Meanwhile, mechanistic studies based on animal models can help analyze and interpret the results of patient-specific CFD studies to facilitate the generation of future predictors.

The unifying hypothesis presented in this review may serve as a starting point to guide the design of these future endeavors. We anticipate that joint effort among clinicians, engineers, and basic scientists focused on better understanding aneurysm pathophysiology will lead to better risk prediction and management of intracranial aneurysms.

\section{ACKNOWLEDGMENTS}

We are grateful for stimulating discussions with Dr Charles Strother, and H.M. is grateful to Dr William L. Young for his mentorship.

Disclosures: Hui Meng—RELATED: Grant: National Institutes of Health/National Institute of Neurological Disorders and Stroke R01NS064592.* Adnan SiddiquiUNRELATED: Board Membership: Codman \& Shurtleff, Comments: Advisory Board; Consultancy: Codman \& Shurtleff, Concentric Medical, ev3/Covidien Vascular Therapies, GuidePoint Global Consulting, Penumbra, Stryker Neurovascular, Employment: University at Buffalo, Kaleida Health, Grants/Grants Pending: National Institutes of Health, University at Buffalo, Payment for Lectures (including service on Speakers Bureaus): Codman \& Shurtleff, Genentech, Stock/Stock Options: Hotspur, Intratech Medical, StimSox, Valor Medical, Travel/Accommodations/Meeting Expenses Unrelated to Activities Listed: Covidien, ${ }^{*}$ MicroVention, ${ }^{\star}$ Other: Penumbra, Comments: serve on National Steering Committee for Penumbra, Inc, 3D Separator Trial (no compensation received). * Money paid to the institution.

\section{REFERENCES}

1. Rinkel GJ, Djibuti M, Algra A, et al. Prevalence and risk of rupture of intracranial aneurysms: a systematic review. Stroke 1998;29:251-56

2. Connolly ES Jr, Rabinstein AA, Carhuapoma JR, et al. Guidelines for the management of aneurysmal subarachnoid hemorrhage: a guideline for healthcare professionals from the American Heart Association/American Stroke Association. Stroke 2012;43:1711-37

3. Wiebers DO, Torner JC, Meissner I. Impact of unruptured intracranial aneurysms on public health in the United States. Stroke 1992;23:1416-19

4. Metaxa E, Tremmel M, Natarajan SK, et al. Characterization of critical hemodynamics contributing to aneurysmal remodeling at the basilar terminus in a rabbit model. Stroke 2010;41:1774-82

5. Xiang J, Natarajan SK, Tremmel M, et al. Hemodynamic-morphologic discriminants for intracranial aneurysm rupture. Stroke 2011;42:144-52

6. Cebral JR, Mut F, Weir J, et al. Quantitative characterization of the 
hemodynamic environment in ruptured and unruptured brain aneurysms. AJNR Am J Neuroradiol 2011;32:145-51

7. Jou LD, Lee DH, Morsi H, et al. Wall shear stress on ruptured and unruptured intracranial aneurysms at the internal carotid artery. AJNR Am J Neuroradiol 2008;29:1761-67

8. Shojima M, Oshima M, Takagi K, et al. Magnitude and role of wall shear stress on cerebral aneurysm: computational fluid dynamic study of 20 middle cerebral artery aneurysms. Stroke 2004;35:2500-05

9. Boussel L, Rayz V, McCulloch C, et al. Aneurysm growth occurs at region of low wall shear stress: patient-specific correlation of hemodynamics and growth in a longitudinal study. Stroke 2008;39:2997-3002

10. Acevedo-Bolton G, Jou LD, Dispensa BP, et al. Estimating the hemodynamic impact of interventional treatments of aneurysms: numerical simulation with experimental validation: technical case report. Neurosurgery 2006;59:E429-30, author reply E429-30

11. Castro MA, Putman CM, Sheridan MJ, et al. Hemodynamic patterns of anterior communicating artery aneurysms: a possible association with rupture. AJNR Am J Neuroradiol 2009;30:297-302

12. Qian $Y$, Takao H, Umezu M, et al. Risk analysis of unruptured aneurysms using computational fluid dynamics technology: preliminary results. AJNR Am J Neuroradiol 2011;32:1948-55

13. Sugiyama SI, Meng H, Funamoto K, et al. Hemodynamic analysis of growing intracranial aneurysms arising from a posterior inferior cerebellar artery. World Neurosurg 2012;78:462-68

14. Takao H, Murayama Y, Otsuka S, et al. Hemodynamic differences between unruptured and ruptured intracranial aneurysms during observation. Stroke 2012;43:1436-39

15. Kallmes DF. Point: CFD—computational fluid dynamics or confounding factor dissemination. AJNR Am J Neuroradiol 2012;33: 395-96

16. Cebral JR, Meng H. Counterpoint: realizing the clinical utility of computational fluid dynamics-closing the gap. AJNR Am J Neuroradiol 2012;33:396-98

17. Robertson AM, Watton PN. Computational fluid dynamics in aneurysm research: critical reflections, future directions. AJNR Am J Neuroradiol 2012;33:992-95

18. Ujiie H, Tachibana H, Hiramatsu O, et al. Effects of size and shape (aspect ratio) on the hemodynamics of saccular aneurysms: a possible index for surgical treatment of intracranial aneurysms. Neurosurgery 1999;45:119-30, discussion 129-30

19. Humphrey JD, Taylor CA. Intracranial and abdominal aortic aneurysms: similarities, differences, and need for a new class of computational models. Annu Rev Biomed Eng 2008;10:221-46

20. Nixon AM, Gunel M, Sumpio BE. The critical role of hemodynamics in the development of cerebral vascular disease. J Neurosurg 2010;112:1240-53

21. Malek AM, Alper SL, Izumo S. Hemodynamic shear stress and its role in atherosclerosis. JAMA 1999;282:2035-42

22. Ota R, Kurihara C, Tsou TL, et al. Roles of matrix metalloproteinases in flow-induced outward vascular remodeling. J Cereb Blood Flow Metab 2009;29:1547-58

23. Frosen J, Tulamo R, Paetau A, et al. Saccular intracranial aneurysm: pathology and mechanisms. Acta Neuropathol 2012;123:773-86

24. Wang Z, Kolega J, Hoi Y, et al. Molecular alterations associated with aneurysmal remodeling are localized in the high hemodynamic stress region of a created carotid bifurcation. Neurosurgery 2009;65: 169-77, discussion 177-78

25. Kolega J, Gao L, Mandelbaum M, et al. Cellular and molecular responses of the basilar terminus to hemodynamics during intracranial aneurysm initiation in a rabbit model. J Vasc Res 2011;48:429-42

26. Findlay JM, Hao C, Emery D. Non-atherosclerotic fusiform cerebral aneurysms. Can J Neurol Sci 2002;29:41-48

27. Park SH, Yim MB, Lee CY, et al. Intracranial fusiform aneurysms: It's pathogenesis, clinical characteristics and managements. $J$ Korean Neurosurg Soc 2008;44:116-23
28. Yong-Zhong G, van Alphen HA. Pathogenesis and histopathology of saccular aneurysms: review of the literature. Neurol Res 1990;12:249-55

29. Hashimoto T, Meng H, Young WL. Intracranial aneurysms: links among inflammation, hemodynamics and vascular remodeling. Neurol Res 2006;28:372-80

30. Stehbens WE. Pathology and pathogenesis of intracranial berry aneurysms. Neurol Res 1990;12:29-34

31. Inci S, Spetzler RF. Intracranial aneurysms and arterial hypertension: a review and hypothesis. Surg Neurol 2000;53:530 - 40, discussion 540-42

32. Finlay HM, Whittaker P, Canham PB. Collagen organization in the branching region of human brain arteries. Stroke 1998;29:1595-601

33. Steiger HJ. Pathophysiology of development and rupture of cerebral aneurysms. Acta Neurochir Suppl (Wien) 1990;48:1-57

34. Aoki T, Kataoka H, Morimoto M, et al. Macrophage-derived matrix metalloproteinase- 2 and -9 promote the progression of cerebral aneurysms in rats. Stroke 2007;38:162-69

35. Hashimoto N, Handa H, Nagata I, et al. Animal model of cerebral aneurysms: pathology and pathogenesis of induced cerebral aneurysms in rats. Neurol Res 1984;6:33-40

36. Meng H, Metaxa E, Gao L, et al. Progressive aneurysm development following hemodynamic insult. J Neurosurg 2011;114:1095-103

37. Meng H, Wang Z, Hoi $Y$, et al. Complex hemodynamics at the apex of an arterial bifurcation induces vascular remodeling resembling cerebral aneurysm initiation. Stroke 2007;38:1924-31

38. Cai J, He C, Yuan FL, et al. A novel haemodynamic cerebral aneurysm model of rats with normal blood pressure. J Clin Neurosci 2012;19:135-38

39. Meng $\mathrm{H}$, Xiang J, Liaw $\mathrm{N}$. The role of hemodynamics in intracranial aneurysm initiation. Int Rev Thromb 2012;7:40-57

40. Juvela $S$. Natural history of unruptured intracranial aneurysms: risks for aneurysm formation, growth, and rupture. Acta Neurochir Suppl 2002;82:27-30

41. Vega C, Kwoon JV, Lavine SD. Intracranial aneurysms: current evidence and clinical practice. Am Fam Physician 2002;66:601-08

42. Cebral JR, Raschi M. Suggested connections between risk factors of intracranial aneurysms: a review. Ann Biomed Eng 2013;41:1366-83

43. Dolan JM, Kolega J, Meng H. High wall shear stress and spatial gradients in vascular pathology: a review. Ann Biomed Eng 2013;41:1411-27

44. Aoki T, Kataoka H, Shimamura M, et al. Nf-kappaB is a key mediator of cerebral aneurysm formation. Circulation 2007;116:2830-40

45. Jamous MA, Nagahiro S, Kitazato KT, et al. Vascular corrosion casts mirroring early morphological changes that lead to the formation of saccular cerebral aneurysm: an experimental study in rats. J Neurosurg 2005;102:532-35

46. Chalouhi N, Ali MS, Jabbour PM, et al. Biology of intracranial aneurysms: role of inflammation. J Cereb Blood Flow Metab 2012; 32:1659-76

47. Asari S, Ohmoto T. Long-term outcome of surgically treated unruptured cerebral aneurysms. Clin Neurol Neurosurg 1994;96:230-35

48. Inagawa $T$, Hirano A. Autopsy study of unruptured incidental intracranial aneurysms. Surg Neurol 1990;34:361-65

49. Mizoi K, Yoshimoto T, Nagamine Y. Types of unruptured cerebral aneurysms reviewed from operation video-recordings. Acta Neurochir 1996;138:965-69

50. Kadasi LM, Dent WC, Malek AM. Cerebral aneurysm wall thickness analysis using intraoperative microscopy: effect of size and gender on thin translucent regions. J Neurointerv Surg 2013;5:201-06

51. Chyatte D, Bruno G, Desai S, et al. Inflammation and intracranial aneurysms. Neurosurgery 1999;45:1137-46, discussion 1146-47

52. Frösen J, Piippo A, Paetau A, et al. Remodeling of saccular cerebral artery aneurysm wall is associated with rupture: histological analysis of 24 unruptured and 42 ruptured cases. Stroke 2004;35:2287-93

53. Kataoka K, Taneda M, Asai T, et al. Structural fragility and inflammatory response of ruptured cerebral aneurysms: a comparative study between ruptured and unruptured cerebral aneurysms. Stroke 1999;30:1396-401 
54. Kataoka K, Taneda M, Asai T, et al. Difference in nature of ruptured and unruptured cerebral aneurysms. Lancet 2000;355:203-03

55. Reynolds MR, Willie JT, Zipfel GJ, et al. Sexual intercourse and cerebral aneurysmal rupture: potential mechanisms and precipitants. J Neurosurg 2011;114:969-77

56. Jiang J, Strother C. Computational fluid dynamics simulations of intracranial aneurysms at varying heart rates: a "patient-specific" study. J Biomech Eng 2009;131:091001

57. Foutrakis GN, Yonas H, Sclabassi RJ. Saccular aneurysm formation in curved and bifurcating arteries. AJNR Am J Neuroradiol 1999; 20:1309-17

58. Tremmel M, Dhar S, Levy EI, et al. Influence of intracranial aneurysm-to-parent vessel size ratio on hemodynamics and implication for rupture: results from a virtual experimental study. Neurosurgery 2009;64:622-30, discussion 630-31

59. Bian C, Xu G, Wang JA, et al. Hypercholesterolaemic serum increases the permeability of endothelial cells through zonula occludens-1 with phosphatidylinositol 3-kinase signaling pathway. J Biomed Biotechnol 2009;2009:814979

60. Ross R, Glomset JA. The pathogenesis of atherosclerosis (first of two parts). N Engl J Med 1976;295:369-77

61. Galis ZS, Sukhova GK, Lark MW, et al. Increased expression of matrix metalloproteinases and matrix degrading activity in vulnerable regions of human atherosclerotic plaques. J Clin Invest 1994;94: 2493-503

62. Chiu JJ, Chien S. Effects of disturbed flow on vascular endothelium: pathophysiological basis and clinical perspectives. Physiol Rev 2011;91:327-87

63. Ross R. Mechanisms of disease: atherosclerosis—an inflammatory disease. N Engl J Med 1999;340:115-26
64. Meng H, Wang Z, Kim M, et al. Saccular aneurysms on straight and curved vessels are subject to different hemodynamics: implications of intravascular stenting. AJNR Am J Neuroradiol 2006;27:1861-65

65. Dhar S, Tremmel M, Mocco J, et al. Morphology parameters for intracranial aneurysm rupture risk assessment. Neurosurgery 2008; 63:185-96, discussion 196-97

66. Baharoglu MI, Schirmer CM, Hoit DA, et al. Aneurysm inflow-angle as a discriminant for rupture in sidewall cerebral aneurysms: morphometric and computational fluid dynamic analysis. Stroke 2010;41:1423-30

67. Honda HM, Hsiai T, Wortham CM, et al. A complex flow pattern of low shear stress and flow reversal promotes monocyte binding to endothelial cells. Atherosclerosis 2001;158:385-90

68. Horie N, Morikawa M, Fukuda S, et al. Detection of blood blisterlike aneurysm and intramural hematoma with high-resolution magnetic resonance imaging. J Neurosurg 2011;115:1206-09

69. Michel JB. Anoikis in the cardiovascular system: known and unknown extracellular mediators. Arterioscler Thromb Vasc Biol 2003;23:2146-54

70. Gui T, Shimokado A, Sun Y, et al. Diverse roles of macrophages in atherosclerosis: from inflammatory biology to biomarker discovery. Mediators Inflamm 2012;2012:693083

71. Zeng Z, Kallmes DF, Durka MJ, et al. Sensitivity of CFD based hemodynamic results in rabbit aneurysm models to idealizations in surrounding vasculature. J Biomech Eng 2010;132:091009

72. Dolan JM, Meng H, Sim FJ, et al. Endothelial cells express a unique transcriptional profile under very high wall shear stress known to induce expansive arterial remodeling. Am J Physiol Cell Physiol 2012; 302:C1109-18 